whole, it may be said that while we are fairly clear as to the nature of the genetical differences between related species, and it has been possible to imitate them within a single species (for example, by producing races which differ morphologically and will not cross), we are only rarely able to say with any certainty how species have originated in Nature.

The study of populations has developed a mathematical theory, due largely to Wright, Fisher, Bernstein, Haldane and Norton. The most important problems studied have been the effects on a population of inbreeding, selection, and mutation, and the theory of estimation from samples. These involve among other things nonlinear integrals and finite difference equations, and complex theorems in inverse probability. Their general result may be said to favour a modified Darwinian theory of evolution.

It must be emphasised that the main bulk of genetical research has been done by authors not named in this summary, and even where no new principles have been discovered, their work has shown that the general laws laid down by Mendel have as wide a validity for genetics as have Dalton's for chemistry.

\title{
Nuclear Structure and Chromosomes
}

\section{By Prof. R. Ruggtes Gates, F.R.S., Professor of Botany, University of London}

$\mathrm{T}$ HE quarter-century of the King's reign has seen many striking advances in cytology, and particularly in our knowledge of the structure of the nucleus and its chromosomes. During the decade ending in 1910, the general theory of 'individuality' of the chromosomes had been established by the work of Boveri and others, the sex chromosomes had been discovered by McClung, and their general relation to sex determined, chiefly by the work of Wilson and his school. These remarkable beginnings definitely linked cytology with genetics and added strength to the view, already accepted in many quarters, that the chromosome reduction in meiosis furnished the physical basis for Mendelian segregation.

An equally notable line of research begun before 1910 was in determining the cytological basis of the mutations discovered by de Vries in Oenothera. This work laid the foundation for an analysis of mutations, so that by 1915 it was recognised that each mutation was in effect a cell change handed on by mitosis to every cell of the mutant. It was also recognised at this time that mutations could be classified into various types, which depended for their origin upon different kinds of chromosome change. The process afterwards known as non-disjunction had been discovered in the pollen mother-cells of Oenothera rubrinervis in 1908, and by 1912 it was proved that the mutant Oe. lata must have arisen by such a process, as it possessed an extra chromosome. Many other Oenothera mutations are now known to have an extra chromosome, and the conception of parallel mutations was founded upon the occurrence of lata and other mutations in different species.

These chromosomal mutations were regarded by many as convincing evidence of the chromosome theory of heredity. Recently (unpublished), trisomic $(2 n+1)$ mutations have been found occurring on a large scale in a wild species of Oenothera. Similar conditions have been observed by Huskins (1927) in fatuoid oats and speltoid wheats, plants with $40,41,42,43$ and 44 chromosomes being found where 42 is the normal number, but the exact relation between fatuoidy and the extra chromosome is still undecided. Blakeslee and his colleagues have recognised the twelve trisomic mutants to be expected in Datura, which has twelve pairs of chromosomes, as well as a series of secondary forms derived from exchange of segments in the primary trisomics.

In 1907-9 the mutant Oenothera gigas was recognised as a cell giant which had doubled its chromosomes. This was the beginning of the enormous modern field of polyploidy, or plants with chromosome multiples. The mutant semigigas was found to have 21 chromosomes (triploid) by Stomps and Lutz independently in 1912. Triploidy was discovered in Drosophila in 1921 and in Datura in the following year. While rare in animals, polyploidy is so widespread in flowering plants that a genus which does not show at least one case of it may almost be regarded as exceptional. Nearly all our cultivated plants, such as wheat, oats, sugarcane, cotton, apples, cherries, tomatoes, potatoes and pineapples, are now known to have varieties or species which possess different multiples of a particular basic number. Among wild species of roses, chrysanthemums, maples, horse-chestnuts, docks, violets, primroses, clovers, ragweeds, strawberries and many others, similar conditions prevail, so that the evolution of many plant genera has clearly been accompanied by the development of higher chromosome multiples from an original basic number, such as $b=7,9,13$ or 17 , for the genus. Where $2 b$ is the ordinary (diploid) condition, some species may have $4 b, 5 b$, $6 b, 10 b$, or occasionally even higher multiples. 
Thus in the genus Potentilla, where $b=7$, species with $2 b, 4 b, 8 b, 10 b, 12 b, 14 b$ and $16 b$ are known.

In the cottons, where $b=13$, it was found (Denham, 1924) that the Old World species had $2 b$ chromosomes while the cultivated American cottons have $4 b$. More recently, several wild species in Lower California and the Galapagos have been found to be diploid. When and how the chromosome doubling in the cultivated cottons took place is at present unknown.

Wild species with an odd number of chromosome multiples reproduce apomictically, as, for example, the pentaploid roses, the tiger lily, which is triploid, and the triploid day lilies. In large genera there may be several basic numbers, as in Primula, where different sections or subsections of the genus have $9,10,11$ and 12 respectively as basic numbers. Less is known as to how one basic number changes into another, but there is evidence that end-toend fusion of certain chromosomes, fragmentation and non-disjunction as well as other changes have been at work. In Drosophila it seems clear that six pairs of chromosomes, which some species (for example, $D$. virilis) still have, was the original number, this number being diminished to five pairs in D. obscura and four in D. melanogaster and other species by fusion to form the long pairs. $D$. Willistoni has only three pairs, the small pair having disappeared. Changes in the relative lengths of chromosomes have also been taking place in various genera.

These discoveries regarding polyploidy are fundamental for an understanding of phylogeny, the results of crossing, and various other fields of genetics.

In 1922 Blakeslee discovered haploidy in Datura, that is, that an egg cell may develop parthenogenetically to produce a plant having a single set of chromosomes in its nuclei. Such a plant is usually much dwarfed and almost com. pletely sterile, since the chromosomes have no mates and are irregularly distributed in meiosis ; but it is significant that it has the morphology of the sporophyte. Haploids have since been discovered in such plants as Crepis, tobacco, wheat, tomatoes, Oenothera, and in rice, where Japanese investigators have found them to occur with exceptional frequency.

Finally may be mentioned the remarkable cases of amphidiploidy, in which a sterile interspecific hybrid doubles its chromosomes and so becomes in effect a new fertile species with a higher chromosome number. The early case of Primula kewensis is now known to be of this character (Pellew and Newton, 1929). Nicotiana digluta was produced in this way by Goodspeed and Clausen in 1925 and Digitalis mertonensis at Merton in 1928. There is evidence that such cultivated plants as tobacco and the loganberry have originated by similar processes. Remarkable in this connexion is the history of Dahlia variabilis, which is native to Mexico and shows a double series of colours as well as the well-known striking morphological variations. It has been shown to be an amphidiploid, the two colour series being contributed by different species (Lawrence, 1929). It may well have originated under cultivation in Aztec gardens.

Even more striking has been the creation of the 'new genus' Raphanobrassica by crosses between the cabbage and the radish, each with $n=9$ chromosomes (Karpechenko, 1924), and Aegilotrichum by crosses between Aegilops and wheats (Tschermak and Bleier, 1926). Amphidiploids between wheat and rye have also been obtained by Tumyakov at the Saratov station on the Volga, and recently by Lebedeff in the Ukraine, who claims that they reproduce apomictically. If so, this is a further similarity to some of our wild polyploid species in such genera as Hieracium and Antennaria. The fact that the tetraploid hemp nettle, Galeopsis Tetrahit, a well-known Linnean species in the British flora, has been synthesised by crossing two related diploid species, and that the rice grass, Spartina Townshendii, which spreads so rapidly in coastal waters, was found to be an amphidiploid hybrid between a British and an introduced American species, shows the importance of polyploidy in phylogeny and in connexion with taxonomic studies. The experimental amphidiploids are new species in every sense of the word, for not only do they breed true in the main, like other species, but they are partially sterile with the species which produced them. The two processes of doubling the chromosome sets and then differentiating them through gene mutations can be traced in many plant genera.

To return to 1910 : Morgan published his first mutation in Drosophila-red eyes to white-in that year, and in the following year found nine wing mutations and five mutations in eye colour. In 1912 the study of sex-linkage in Drosophila began, crossing-over soon came to be studied on an unprecedented scale and in this way more than five hundred mutations have been assigned their relative positions in the four pairs of chromosomes. By 1926 it was possible for Morgan to announce his law that the number of linkage groups corresponds with the number of chromosome pairs. This was confirmed by Punnett for Lathyrus in 1927 ; and in maize, where each of the ten pairs of chromosomes can be identified by its appearance, a map of the genes in each chromosome has been constructed by crossing-over experiments, chiefly by the Cornell school. In 1916, Bridges used cases of non-disjunction of the $X$-chromosomes in Drosophila to prove that sex-linked genes were 
borne in the $X$. Later, he also found individuals with a third member of the tiny fourth chromosome, as well as others having but one member of this pair.

Morgan's theory of crossing-over was founded upon Janssen's observations (1909) of chiasmata in meiosis, but exactly how chiasmata are formed and how they are related to crossing-over is still a matter of controversy on which many observations are being made and various views are held.

In $1922, \mathrm{~L}$. V. Morgan found a strain of Droso= phila in which the two $X$-chromosomes of the female had become permanently attached end-toend. Before the condition was observed cytologically it had been predicted on the basis of the peculiar breeding results with this strain. The condition has since appeared independently in other strains.

Arrangement of the chromosomes of Oenothera in a chain at diakinesis was discovered in 1908, and in 1922 Cleland found that the number connected into a ring was characteristic of certain species. This condition, which is now known as catenation, is recognised as fundamental to the genetics of Oenothera, and the catenation in many species, hybrids and mutations, has been determined. It accounts for the usual absence or infrequent occurrence of ordinary Mendelian segregation, the occurrence of 'complexes' of characters, the twin hybrids of de Vries and the fact that heterozygous species breed true. In order to account for all these phenomena it is necessary to assume, not only that the chromosomes occupy fixed positions in the ring, but also that they have a fixed orientation. This has recently been shown experimentally to be the case. The 14 chromosomes of Oenothera may be all in a ring or in seven pairs or with various arrangements of smaller rings. The fifteen possible groupings (a few unpublished) have now all been observed in different forms of Oenothera.

Chromosome catenation has since been discovered in a number of other plant genera, including Datura, Aucuba, Rhoeo and Pisum. It can be produced by crossing two Oenotheras each with seven free pairs, and also by crossing certain strains of Datura or Pisum. Of special interest is the cross between a Tibetan and a European variety of Pisum (Pellew and Sansome, 1931) in which a ring of four chromosomes arises. Catenation has also recently been produced in Oenothera by exposing the pollen to X-rays. The theory of segmental interchange was proposed by Belling and Blakeslee (1926) to account for the chromosome linkage arising in certain Datura crosses, and has since been shown to be widely applicable to Oenothera and all other cases in which ring formation takes place as a result of crossing.
In 1927 Muller announced that the mutation rate in Drosophila could be increased a hundredfold by subjecting the germ cells to $\mathrm{X}$-rays. The exact nature of the effect on the chromosomes is still unknown, but in addition to gene mutations, many of which are lethal, chromosome fragmentations, deletions and segmental translocations are produced by this means. It has been applied to various plants, notably Nicotiana, barley, Antirrhinum and recently Oenothera. There are now many lines of evidence that translocation of chromosome segments can take place in Nature, and it has been shown, for example, by comparing the seriation of the genes in Drosophila melanogaster with that in D. simulans, that a segment of chromosome III in one of these species has been inverted.

Phylogenetic significance also attaches to the secondary pairing of chromosome bivalents in meiosis. This was first observed by the Marchals (1911) in mosses in which the chromosome numbar had been doubled experimentally. Hagerup (1927) noted it in a hermaphrodite tetraploid species evidently derived from Empetrum nigrum. Darlington (1928) used it in interpreting the history of the polyploid cherries. Secondary pairing appears to be due to a residual attraction, and indicates that the chromosome bivalents which show it are themselves more distantly homologous. In other words, it is an indication of polyploidy or similar processes in the more remote ancestry, and throws light on how chromosome numbers have changed from genus to genus. The Pomoideæ, a group of Rosaceous genera, have 17 chromosomes. They show secondary pairing and other multivalent associations (Darlington and Moffatt, 1930) which indicate how this number has been derived from an earlier 7, which is characteristic of many genera in the family, such as Rosa itself. Secondary pairing has been observed also in Pyrus, Dahlia, Brassica, Gossypium and many other cases, where it helps to throw light on nuclear phylogeny (Lawrence, 1931).

Important advances have also taken place in regard to chromosome structure, although in this fascinating field many points are still unsettled. Most investigators appear to be now agreed that the chromosomes are double in anaphase as well as prophase, from which it follows that the split occurs about the time of metaphase and that the chromosome is a double structure throughout the mitotic cycle. The chromonema theory, according to which the essential part of the chromosome is a thread of uniform thickness, dates from Vejdovsky, 1912. The chromomere theory, of granules aligned like beads on a string, is much older. Both views have strong supporters at the present time and it does not yet appear how their differences will be 
resolved. Wenrich (1916) found that in grasshoppers particular chromomeres could be identified by their size and position on the chromosome. Belling (1931) identified the chromomeres with the genes, and attempts at counting them in various plants have given values ranging roughly from 1000 to 2500 . The chromosome threads become spiral in various stages of mitosis, and Kuwada has found indications of a spiral within the spiral. Others find the spiral chromonema splitting lengthwise in prometaphase.

In what may be called the external morphology of the chromosomes more marked progress has been made. In 1912, S. Navashin discovered that certain chromosomes in Galtonia have a tiny more or less globularsatellite attached to oneend by a thread. One or more pairs of satellited chromosomes have since been observed in many plants and animals.

The spindle fibre attachment constriction is now a well-recognised feature of all plant chromosomes, and 'kinetic bodies', 'knobs', 'heads', additional constrictions, vesicles and their special features have been observed. Levitsky, M. Navashin and others of the Russian school have been active in comparing the karyotypes of various plant groups.

The nature of the nucleolus has long been a mystery, but light has recently been thrown on this problem. Wenrich (1916) noted in the meiotic nuclei of a grasshopper that the nucleolus bore a constant relation to a particular pair of chromosomes. Later a definite 'nucleolar body' was discovered as a darker staining area of the nucleolus in pollen mother cells of Lathyrus (Latter, 1926), and it was shown that a loop of the chromosome thread was constantly attached to it. The same condition has since been found in Lathroea, Oenothera, Malva and rice, and frequently two such bodies rather than one are attached to the nucleolus. S. Navashin had observed a pair of satellites attached to the nucleolus, and in studies of maize, McClintock (1931) has shown that the satellited pair of chromosomes is concerned in producing the nucleolus in teleophase at a particular locus. When these chromosomes are widely separated, two nucleoli will be produced. Heitz (1931) has observed similar conditions in Vicia and other plants as well as in insects (1933), while Dearing (1934) shows in the amphibian Amblystoma the same relation between a satellited pair of chromosomes and the (usually two) nucleoli present. Thus it appears to be the function of a particular locus of one pair of chromosomes to produce the nucleolus, but this is not the whole story. In this, as in other fields of nuclear study, the results in animals and plants have been re. markably synchronised.

Lack of space prevents more than mention of the important method of micro-dissection, by which living cells can be dissected with glass needles under an immersion lens. Introduced by Kite and Chambers in 1912, it has led to many interesting observations of the physical condition of various cell constituents, including the chromosomes.

Finally, we may refer to the investigations of the chromosome structure in the salivary glands of insects. These giant chromosomes have long been known to show a banded structure, but its significance has only been brought out by the recent observations of Painter, Bridges, Koltzoff and others. The discs differ markedly in details of structure, but they are definitely spaced at varying intervals along the chromosomes, and from the evidence of their pairing and genetio behaviour it is clear that they must be identified in some way with the genes. Certain of the bands have been shown to vary according to the genetic make-up of the animal. Bridges has just published $(J$. Hered., 26, No. 2) maps of the four chromosomes (significantly separated into six) in the salivary glands of Drosophila melanogaster, identifying each band and also pointing out many other structural features or landmarks at various loci of the chromosomes. These chromosomes total about $1180 \mu$, or 150 times the length of the meiotic chromosomes. The bands, which probably represent loci, number 2650 , the little fourth chromosome having 34 . This begins a new era in chromosome study.

\section{Chemistry of the Anthocyanins}

\section{By Prof. R. Robinson, F.R.s., Waynflete Professor of Chemistry, University of Oxford}

$\mathrm{T}$ Elassical paper of Willstätter and Everest (1913), on the isolation of the pigment of blue cornflowers, heralded a dramatic transformation of the state of our knowledge of the blue and red colouring matters of flowers and blossoms, and the present position is that we not only know the molecular structure of the more important and widespread anthocyanins, but also that many of them have been made artificially in the laboratory. With the simultaneous growth of precise information about chlorophyll, the carotenoids, the polysaccharides and the terpenes, one may say that all the more obvious challenges of vegetative Nature to the organic chemist have been taken up, and taken up successfully. Deep mysteries there are still, it is true, but one must probe 